\title{
Retour sur la fondation de l'Abbaye du Mont-Saint- Michel et le rôle du duc Richard Ier de Normandie
}

\author{
George N. Gandy
}

\section{(2) OpenEdition}

1 Journals

Édition électronique

URL : http://journals.openedition.org/abpo/3202

DOI : $10.4000 / a b p o .3202$

ISBN : 978-2-7535-5040-7

ISSN : 2108-6443

Éditeur

Presses universitaires de Rennes

Édition imprimée

Date de publication : 22 avril 2016

Pagination : 7-33

ISBN : 978-2-7535-5038-4

ISSN : 0399-0826

\section{Référence électronique}

George N. Gandy, "Retour sur la fondation de l'Abbaye du Mont-Saint-Michel et le rôle du duc Richard ler de Normandie ", Annales de Bretagne et des Pays de l'Ouest [En ligne], 123-1 | 2016, mis en ligne le 22 avril 2018, consulté le 10 décembre 2020. URL : http://journals.openedition.org/abpo/3202 ; DOI : https://doi.org/10.4000/abpo.3202 


\title{
Retour sur la fondation de l'abbaye du Mont-Saint-Michel et le rôle du duc Richard I ${ }^{e r}$ de Normandie
}

\author{
George N. GANDY \\ Historien, juriste (en retraite)
}

\begin{abstract}
L'histoire de la formation du duché de Normandie au cours des $\mathrm{x}^{\mathrm{e}}$ et $\mathrm{XI}^{\mathrm{e}}$ siècles suscite toujours des controverses ${ }^{1}$. Au cours des $\mathrm{IX}^{\mathrm{e}}$ et $\mathrm{X}^{\mathrm{e}}$ siècles, l'interruption de la vie monastique et des successions épiscopales, Rouen mis à part, entraîna aussi la disparition de l'écriture dans la région et par conséquent une quasi-absence de témoignages. De plus, si peu à peu, à partir de la cour ducale, des sources écrites réapparaissent autour de l'an mil, avec l'œuvre de Dudon de Saint-Quentin notamment, les historiens sont mal armés pour évaluer les histoires partisanes qu'elles rapportent à cause de l'obscurité de la période précédente.

Il y a peut-être eu, par conséquent, trop d'empressement à accepter la version normande des événements. C'est à l'évidence le point de vue de quelques historiens contemporains pour qui ces premières écritures déclenchèrent une " réécriture "systématique du passé non écrit du duché naissant, en supprimant ou en ajustant des réalités inopportunes afin d'augmenter la renommée des ducs. Un des résultats les plus convaincants de cette entreprise critique a été l'emploi de sources extérieures à la Normandie pour démontrer que l'élargissement du duché vers l'ouest avait été irrégulier après une première phase assez rapide sous Guillaume Longue Épée (927-942), mais aussi accompagné d'échecs que les sources normandes plus tardives ont niés ou ignorés ${ }^{2}$.
\end{abstract}

1. La relecture et l'établissement définitif de ce texte ont été assurés par Florian Mazel. Le comité de rédaction des $A B P O$ le remercie infiniment d'avoir effectué ce travail. L'auteur est également très reconnaissant à Jean-Luc Leservoisier, qui l'a aidé à produire une première version française de cet article, écrit à l'origine en anglais. Il remercie aussi Linda Kirk, David Bates et Richard Allen pour leurs observations critiques.

2. VAN Torhoudt, Éric, Centralité et marginalité en Neustrie et dans le duché de Normandie, thèse de doctorat, Université de Paris 7, 2008, p. 150-154. 
Néanmoins, une méfiance généralisée envers les sources normandes comporte aussi des risques. Quand manquent des preuves extérieures, ce qui est souvent le cas, la force mais également le danger de cette sorte de remise en cause viennent de la liberté qu'elle accorde de proposer pratiquement n'importe quelle interprétation des textes convenant pour un moment particulier. À titre d'exemple, tout récemment Éric van Torhoudt a suggéré que certaines donations au Mont-Saint-Michel, attribuées par le cartulaire du XII ${ }^{\mathrm{e}}$ siècle à Guillaume Longue Épée, lui étaient prêtées à tort par un abbé du Mont, dans les années 1020. La preuve invoquée est que l'abbé en question était originaire de Jumièges, abbaye où Guillaume avait voulu se retirer comme moine et dont les religieux avaient ensuite fortement promu l'hagiographie du souverain comme prince martyr. C'est une explication possible, mais d'une attribution spécieuse qui, elle-même, n'est pas établie et peut être fausse. En fait, comme Éric van Torhoudt lui-même l'a montré ailleurs, Longue Épée a joui d'un tel succès dans l'ouest du duché qu'on peut supposer qu'il a bien pu faire les dons en question ${ }^{3}$.

En ce qui concerne l'histoire du Mont-Saint-Michel, cet empressement à désavouer le récit normand des événements doit beaucoup à la plaidoirie de Katharine Keats-Rohan. Mais si nous voulons éviter d'ajouter davantage de strates aux mythes d'origine du xI ${ }^{\mathrm{e}}$ siècle, l'essentiel est d'examiner de près les contre-propositions des sceptiques modernes. Tel est l'objectif de cet article.

\section{Le clergé du Mont-Tombe}

Selon une tradition dont il n'y a aucune raison convaincante de douter, vers l'an 709, une communauté dotée de douze clercs fut installée sur l'île du Mont-Tombe - nom du Mont-Saint-Michel jusqu'à la deuxième moitié du $\mathrm{X}^{\mathrm{e}}$ siècle, et souvent plus tard - par Aubert, l'évêque d'Avranches, à 10 kilomètres environ à l'est, et donc en Neustrie, pour prendre en charge une église que celui-ci venait d'y construire en l'honneur de saint Michel. $\mathrm{Au} \mathrm{IX}^{\mathrm{e}}$ siècle, le texte fondateur de la communauté, Revelatio ecclesiae sancti Michaelis, a distingué ces clerici de certains monachi qui, selon le texte, avaient habité le lieu dans une période antérieure ${ }^{4}$.

La fraternité d'Aubert a dû comprendre plusieurs catégories de clercs : certainement des prêtres, mais aussi quelques diacres et sous-diacres, des acolytes et des lecteurs, si bien que la communauté pouvait agir comme son propre séminaire, les membres du clergé progressant à travers les grades d'ordination au moyen d'une sorte de formation interne ${ }^{5}$. Une telle

3. Ibidem, p. 326-327. GANDY, George, "Who built what at Mont Saint Michel during the $10^{\text {th }}$ Century?", Annales de Normandie, 65 (2015), p. 160-161.

4. Revelatio, III.2, VII.2. Toute allusion dans cet article à la Revelatio renvoie à l'édition critique par Pierre Bouet et Olivier Desbordes dans Bouet, Pierre et DEsBordes, Olivier (éd.), Chroniques latines du Mont Saint-Michel (IXe-XII siècle), Caen, Presses Universitaires de Caen, 2009. Pour le texte, voir p. 90-103.

5. L'auteur a beaucoup appris en discutant des clercs et chanoines avec Julia Barrow. Cependant, le professeur Barrow n'a aucune responsabilité dans les propos présentés ici. 
structure, hiérarchisée et quasi-autonome, peut contribuer à expliquer la longévité de la communauté et le fait qu'elle survécut même à la disparition de l'épiscopat local pendant l'époque viking, disparition qui commença après $850^{6}$ et dura jusqu'à 990 environ. Le Mont-Tombe fut peut-être la seule maison religieuse de la province de Rouen à subsister ainsi de façon ininterrompue jusqu'à l'époque normande.

Sa survie, cependant, fut également une conséquence des buts qu'elle visait $^{7}$. Fait important, la marque distinctive d'une communauté cléricale était la célébration des messes, une fonction non permise aux moines. De plus, à l'époque carolingienne et peut-être plus tôt, il est probable que les clercs étaient recherchés par ceux qui voulaient se confesser à eux, encore un rôle que les moines ne pouvaient normalement pas remplir. Par conséquent, une communauté de clercs était mieux adaptée pour desservir le public laïque auquel, par nature, un monastère ne s'intéressait pas; et incontestablement, une raison de la pérennité du Mont-Tombe fut le fait que, depuis au moins le milieu du IX ${ }^{\mathrm{e}}$ siècle, cette communauté veillait sur un lieu de pèlerinage de renommée internationale ${ }^{8}$. Il bénéficiait de la situation privilégiée qu'occupaient ses clercs entre ce monde et l'au-delà, entre les visiteurs de l'île et son patron incorporel, le Gardien du Paradis. Seuls des clercs pouvaient assurer la confession des pénitents et satisfaire la demande de célébration de messes, soit pour les pèlerins eux-mêmes soit pour les défunts. Remplacer le clergé du Mont-Tombe par des moines n'aurait pas été utile à grand chose ${ }^{9}$.

Pourtant, à un certain moment, les clercs furent remplacés par des moines, par des bénédictins introduits en 965 par le duc normand, Richard I ${ }^{\text {er }}$ (942-996), si on prête foi au récit des Normands; par des moines d'origine peut-être bretonne, installés au moins une centaine d'années plus tôt, si on préfère les arguments de Katharine Keats-Rohan. Une troisième possibilité, soutenue par Pierre Bouet, accepte la thèse normande mais suggère que longtemps avant l'arrivée des bénédictins normands, dès la première moitié du IX ${ }^{\mathrm{e}}$ siècle, les clercs " aubertiens " avaient été réformés et transformés en maison de chanoines réguliers.

6. Il y avait toujours un évêque au moment de l'écriture de Revelatio ; sur celle-ci, voir plus bas.

7. Gandy, George, "Dans quel but le Mont Saint Michel a-t-il été fondé? ", Revue de l'Avranchin et du Pays de Granville, 92, 2015, p. 219-234.

8. MussEt, Lucien, "Recherches sur les pèlerins et les pèlerinages en Normandie jusqu'à la Première Croisade ", Annales de Normandie, 12, 1962, p. 128-130.

9. Le nombre de moines prêtres augmenta sensiblement à partir des $\mathrm{IX}^{\mathrm{e}}$ et $\mathrm{X}^{\mathrm{e}}$ siècles alors que la célébration eucharistique devenait de plus en plus centrale dans la liturgie monastique. Mais au temps de la fondation du Mont-Tombe la coutume ancienne régnait toujours, l'avancement à la prêtrise ne se rattachant pas aux fonctions sacramentelles mais plutôt à l'idée du martyr, on le réservait aux moines les plus contemplatifs et ascètes (LECLERCQ, Jean, "On monastic priesthood according to the ancient medieval tradition ", Studia Monastica, 3, 1961, p. 137-155). 
Une datation plus précise de la Revelatio devrait permettre de renoncer à cette hypothèse, car au moment de son écriture la communauté était certainement toujours composée de clerici. L'œuvre narre principalement l'histoire des origines du lieu sacré dans les rêves de l'évêque Aubert. Ici l'auteur, certainement membre de la communauté, a repris une tradition, mais il intervient pertinemment dans le récit, par exemple en faisant des estimations et des comparaisons lorsqu'il décrit les dimensions de l'île ou la forme et la capacité de l'église qu'Aubert y a construite. Par conséquent, son observation selon laquelle l'île sépare l'Avranchin, et ainsi la Neustrie, de la Bretagne doit être correcte ${ }^{10}$.

Comme Nicolas Simonnet l'a fait remarquer le premier, au IX ${ }^{\mathrm{e}}$ siècle le pagus d'Avranches ne fut limitrophe de la Bretagne que pendant une brève période postérieure à 851 , date à laquelle Charles le Chauve céda le comté de Rennes au Breton Erispoë. En 867, la Bretagne gagna aussi le Cotentin, l'Avranchin inclus, et à compter de cette date, la cité épiscopale et le MontTombe lui-même devinrent bretons et le restèrent, au moins théoriquement, jusqu'à la formation de la Normandie au $\mathrm{x}^{\mathrm{e}}$ siècle. Donc, Pierre Bouet mis à part, les historiens contemporains ont en général adopté la conclusion de Simonnet selon laquelle la Revelatio a dû être composée entre 851 et $867^{11}$.

Pierre Bouet a soutenu que d'un point de vue stylistique la rédaction de la Revelatio datait de la première Renaissance carolingienne - il la date des environs de 816 - mais il ne règle pas la question des frontières politiques, et une preuve stylistique est en soi un motif trop imprécis pour dater un texte : ainsi, a-t-elle été écrite par un jeune homme ou par un homme plus âgé qui utilisait toujours le style de sa jeunesse? En outre, Jacques Hourlier, invoquant lui aussi des considérations stylistiques, a conclu que l'œuvre datait de juste après le milieu du IX $\mathrm{x}^{\mathrm{e}}$ siècle ${ }^{12}$. À l'évidence les preuves stylistiques ne sont pas concluantes, et la question des frontières va à l'encontre de l'hypothèse de Bouet, et non de celle d'Hourlier. Nous pouvons donc conclure avec une certaine assurance que la communauté de clergé fondée par Aubert supervisait toujours le lieu sacré en 851.

10. Revelatio, III.1; voir aussi la discussion dans BouEt, Pierre et DESBORDES, Olivier, Chroniques latines..., op. cit., p. 48-49.

11. Simonnet, Nicolas, « La Fondation du Mont-Saint-Michel d'après la Revelatio ecclesiae sancti Michaelis ", Annales de Bretagne et des Pays de l'Ouest, 106, 1999, p. 19. À strictement parler, deux frontières possibles, riveraine ou estuarienne, sont en question : celle qui suivait le Couesnon, coulant vers le nord en se jetant dans la baie du Mont-Saint-Michel tout près du Mont, qui séparait la Neustrie de la Bretagne jusqu'en 851 ; et celle délimitée par la Sélune, qui se jetait dans la baie en coulant d'est en ouest, l'estuaire passant juste au sud de l'île, qui marquait la frontière bretonne en 851-867, après que le Rennais fût passé à la Bretagne. Cependant, comme l'auteur de la Revelatio, toujours précis, situe le Mont par rapport à la Sélune et ne fait aucune référence au Couesnon, on peut déduire presque avec certitude qu'il écrivait en 851-867.

12. Bouet, Pierre et Desbordes, Olivier, Chroniques latines..., op. cit., p. 34-39. Cf. HourLIER, Jacques, "Les Sources écrites de l'histoire montoise antérieure à 966 ", dans FOREVILLE, Raymonde (dir.), Millénaire monastique du Mont-Saint-Michel, t. 2, Paris, Lethielleux, 1967, p. $127-128$. 
Il faut noter également qu'une rencontre significative eut lieu en Bourgogne, en 863 quand Odon de Glanfeuil, fuyant les Vikings avec les reliques de saint Maur, rencontra des pèlerins parmi lesquels un certain Pierre, clericus du Mont-Tombe qui revenait d'un voyage de deux ans à Rome et rapportait des vitae manuscrites de saint Bernard et de cinq de ses disciples, dont la vita de saint Maur lui-même, dont Odon, " pour pas peu de pièces » le persuada de se séparer ${ }^{13}$. Pierre était certainement un prêtre : la communauté n'aurait pas confié une telle mission à un subordonné. Comme il ne pouvait guère rentrer au Mont les mains vides, on peut déduire qu'il avait acquis en plus d'autres manuscrits. Ceci, comme le caractère nonpatristique d'une partie au moins de l'ensemble des documents qu'il rapporta, nous laisse entendre que la communauté insulaire possédait déjà une importante bibliothèque, que ses membres étaient bien instruits et intellectuellement curieux et qu'ils étaient, en outre, globalement assez riches. Qu'ils aient pu laisser partir un prêtre pendant deux ans suggère aussi qu'il y en avait d'autres sur l'îlot et ainsi, même si ce n'est pas une preuve définitive, qu'au moment où Pierre partit, en 861, le groupe qu'il laissa derrière lui était encore une communauté de clercs.

\section{La version normande et ses critiques}

L'histoire normande du Mont-Saint-Michel commence avec Dudon de Saint-Quentin, dont l'histoire des premiers ducs a vraisemblablement été réalisée entre 1015 et $1026^{14}$. Dudon nous dit que Richard ${ }^{\mathrm{er}}$ y construisit " une église d'une ampleur stupéfiante " et divers bâtiments conventuels mais, les preuves archéologiques ne confirmant pas la première partie de cette affirmation, on pourrait en conclure que Dudon donne plus d'arguments aux sceptiques qu'à la cause normande qu'il voulut servir ${ }^{15}$.

Le récit de leurs origines par les moines eux-mêmes commence avec une brève série d'annales composée en 1035-1048, qui, pour l'an 965, atteste en latin : " Il a été autorisé par l'acte du pape Jean et de Lothaire roi des Francs que l'église du Mont-Saint-Michel soit dotée à perpétuité d'un ordre monastique ${ }^{16} . .$. " Une génération plus tard, l'Introductio monachorum, l'histoire de

13. Lettre d'Odon de Glanfeuil à Adelme : Adhelmi Opera dans : EhwaLd, R. (éd.), Monumenta Germaniae Historica, Scriptores, Auctores Antiquissimi. t. 15.1, Berlin, Weidmann, 1919, p. 462-463.

14. NeveuX, François, La Normandie des ducs aux rois, $X^{e}-$-XI ${ }^{e}$ siècle, Rennes, Ouest-France, 1998, p. 17.

15. DudON DE SAINT-QUENTIN, De moribus et actis primorum Normanniae ducum, dans : LAIR, Jules (éd.), Caen, Société des antiquaires de Normandie, 1865, p. 290 (cf. Gandy, George, "Who built what...?", art. cit., p. 165-166).

16. British Library Royal, $13 \mathrm{~A}$ xxIII, $\mathrm{f}^{\circ} 96 \mathrm{r}^{\circ}$, imprimé dans The Cartulary of the abbey of Mont-Saint-Michel, Keats-Rohan, Katharine (éd.), Donington, Shaun Tyas, 2006, p. 187. La dernière entrée a lieu en 1035. Keats-Rohan a daté ces annales de 1035-1040 sur la supposition, sûrement erronée, que si elles avaient été continuées elles auraient noté la mort du comte Alain de Bretagne en 1040. Il est certain, par contre, qu'elles auraient mentionné la démission de l'abbé Suppo du Mont en 1048. 
l'abbaye écrite par les moines vers 1080-1085, présente de cet événement une version beaucoup plus élaborée, qu'on discutera plus tard. Selon celleci, Richard I Ir fut informé que la communauté religieuse insulaire, qui était toujours composée de clercs, bien que l'Introductio les appelle canonici, était tombée dans une telle décadence qu'en 965, le duc se sentit obligé de l'expulser du Mont et d'installer à sa place, avec l'appui d'un diplôme de Lothaire et d'une bulle du pape, des moines bénédictins dont l'abbé se nommait Mainard ${ }^{17}$. À la fin du XI ${ }^{\mathrm{e}}$ siècle, l'abbaye bénédictine se revendiquait donc comme une fondation purement normande.

Pendant longtemps, les historiens eurent tendance à admettre ce récit, même si deux aspects sont restés gênants. D'abord, à la suite de Louis Halphen et Ferdinand Lot, les spécialistes ont toujours considéré la bulle pontificale comme un faux du XI ${ }^{\mathrm{e}}$ siècle ${ }^{18}$. Cela n'est pas fatal au récit de la fondation en général car le seul apport de la bulle à la narration se trouve dans sa prétendue confirmation des droits d'élection revendiqués par les moines ${ }^{19}$ mais, pour certains, cette bulle douteuse jette aussi un doute sur le rôle de Lothaire. Ensuite, l'année 965 paraît bien précoce pour que Richard I ${ }^{\text {er }}$ ait fondé une abbaye si loin de Rouen, dans une région dont certains doutent qu'il l'ait même jamais contrôlée à une quelconque date de son règne. Il n'est donc pas surprenant que la "version normande " ait été contestée, ni que les prétentions de régions voisines au sujet des origines de l'abbaye aient commencé à se faire jour.

Une autre histoire crédible pourrait émerger d'une exploration des liens pré-normands entre le Mont-Saint-Michel et la Bretagne, lesquels, au vu de l'origine des donations faites au monastère dès que des témoignages écrits sont disponibles, étaient assurément forts, en particulier à la fin du $\mathrm{x}^{\mathrm{e}}$ siècle, plusieurs décennies après le prétendu acte de fondation de Richard ${ }^{\text {er }}$ et à une époque où ni lui ni sa famille ni son aristocratie n'avaient fait un seul don de terre à l'abbaye. De même, les liens entre le Mont et le Maine, au sud-est de l'Avranchin, étaient aussi étroits à la fin du $\mathrm{x}^{\mathrm{e}}$ siècle, et nous offrent une autre possibilité.

Cependant, en examinant ces liens, il nous faut tenir compte de ce qui est arrivé aux clercs avec lesquels nous avons commencé. Dans l'Introductio, les clercs que les Normands rencontrèrent sur l'île sont appelés canonici. Pierre Bouet, frappé par ce terme, soutient l'hypothèse que la communauté aubertienne avait dû être transformée en communauté de chanoines vers les années 820 , après avoir adopté la règle promulguée

17. Introductio, VI-VIII. Toute allusion à l'Introductio renvoie à l'édition critique par Pierre Bouet et Olivier Desbordes dans Bouet, Pierre et Desbordes, Olivier, Chroniques latines..., op. cit., Pour le texte, voir p. 202-223.

18. Recueil des actes de Lothaire et de Louis V, rois de France (954-987), HALPHEN, Louis et Lot, Ferdinand (éd.), Paris, Imprimerie Nationale, 1908, numéro 24, p. 53-54; KeATs-RoHAN, Katharine, Cartulary..., op. cit., p. 18-21.

19. Pour le texte, voir Keats-Rohan, Katharine, Cartulary..., op. cit., p. 74. 
en 816-817 à Aix ${ }^{20}$. Bouet admet qu'il n'y a aucune preuve qu'une telle réforme ait eu lieu, et celle-ci est très peu probable. Les termes clerici et canonici, quand ils sont appliqués à des groupes, étaient tout simplement interchangeables à cette période ${ }^{21}$. Ainsi l'usage du mot canonici dans l'Introductio ne signifie pas qu'on a nécessairement réformé les clercs. En deuxième lieu, les réformes canoniales promulguées à Aix avaient pour but principal de créer un corps d'élite de prêtres, vivant en communauté avec leur évêque, qui par leur érudition et leur chasteté pourraient être un modèle pour les simples prêtres moins disciplinés qui servaient le peuple de la cité épiscopale ${ }^{22}$. Cette réforme n'aurait eu aucune pertinence évidente pour une communauté insulaire isolée, à une demi-journée de route de son évêque, dont les membres n'étaient entourés ni d'une population nombreuse à soutenir, ni d'un corps de prêtres à inspirer par leur exemple. De plus, puisque la Revelatio fait référence aux clerici et non aux canonici, elle devrait ainsi dater d'avant la réforme postulée par Bouet, et son hypothèse le pousse, comme nous l'avons vu, à une datation de la Revelatio trop précoce.

Néanmoins, si les clercs d'Aubert ne devinrent pas des chanoines réguliers, d'autres moyens de s'en débarrasser ont été échafaudés. Katharine Keats-Rohan a affirmé que Pierre Bouet avait raison de déterminer le moment d'une réforme des clercs mais qu'il avait tort en suggérant que son but était l'institution de chanoines réguliers. En effet, en 816-817, une autre réforme émanant d'Aix avait tenté d'imposer la règle de saint Benoît à tous les monastères de l'Empire. Katharine Keats-Rohan a soutenu qu'entre les années 820 et 860 , les clercs aubertiens avaient dû devenir, ou faire place non pas à des chanoines mais à des moines bénédictins ${ }^{23}$. Si ceci est vrai, cela renforcerait ses arguments niant qu'il ait pu y avoir une réforme sous Richard I ${ }^{\mathrm{er}}$ pendant les années 960 - ce qu'elle appelle " the reformation myth " des Normands ${ }^{24}$ - car la communauté devait déjà être bénédictine depuis longtemps.

Même si, comme Pierre Bouet, Katharine Keats-Rohan ne peut donner aucune preuve directe d'une réforme bénédictine précoce sur l'île, elle utilise les nécrologes plus tardifs de l'abbaye, ainsi qu'une remarque de Bernard, un moine de passage vers 870 , pour suggérer que celle-ci avait dû avoir eu lieu. Le nécrologe du Mont le plus riche enregistrait les noms des individus, laïques ou religieux, pour les âmes desquels les moines offraient chaque année des prières lors de l'anniversaire de leur décès. La version conservée à l'heure actuelle, contenant plus de 7000 noms, fut rédigée au

20. Bouet, Pierre et Desbordes, Olivier, Chroniques latines..., op. cit., p. 35-39.

21. Julia Barrow, courriel à l'auteur, 24 juin 2013.

22. Claussen, M. A., The Reform of the Frankish Church: Chrodegang of Metz and the "Regula canonicorum " in the Eighth Century, Cambridge, Cambridge University Press, 2004, p. 12-15.

23. Keats-Rohan, Katharine, Cartulary..., op. cit., p. 14-17.

24. Ibidem, p. 21. 
début du XIII ${ }^{\mathrm{e}}$ siècle à partir de matériaux plus anciens ${ }^{25}$. L'historien bénédictin Jean Laporte a pu identifier provisoirement un certain nombre de ces personnes, dont neuf sont décédées avant 965 , les deux premières respectivement en 798 et 841, ce qui démontre, selon Katharine Keats-Rohan, que le monachisme aurait été présent au Mont de manière ininterrompue pendant une grande partie, voire la totalité du $\mathrm{IX}^{\mathrm{e}}$ siècle $^{26}$.

Pourtant, le nécrologe ne permet en rien une telle conclusion. 125 individus y ont été provisoirement identifiés entre la fin du viII ${ }^{\mathrm{e}}$ et la fin du $\mathrm{XII}^{\mathrm{e}}$ siècle, dont 115, c'est-à-dire $92 \%$, sont décédés après l'an mil. En suivant Katharine Keats-Rohan, il apparaît qu'au x ${ }^{\mathrm{e}}$ siècle dans sa totalité seulement trois moines identifiables ont été commémorés ${ }^{27}$. Il est beaucoup plus probable que la compilation du nécrologe n'a commencé que vers l'an mil, même si quelques personnes, mortes depuis longtemps mais toujours vénérées, y furent alors incluses. Des nécrologes du même genre que celui du Mont-Saint-Michel apparaissent ailleurs à peu près à la même époque, à partir de la deuxième moitié du $\mathrm{x}^{\mathrm{e}}$ siècle, et constituent un trait commun de la culture monastique du début du XI ${ }^{\mathrm{e}}$ siècle ${ }^{28}$. Si, contrairement aux tendances générales, cette forme de commémoration avait été instituée au Mont-Saint-Michel au début du $\mathrm{x}^{\mathrm{e}}$ siècle, il faudrait expliquer non seulement une telle précocité mais aussi pourquoi la pratique y évolua si lentement par la suite; pourquoi, autrement dit, les noms qui apparaissent dans la liste pendant les deux premières centaines d'années de sa supposée existence sont si peu nombreux. En fait, le nécrologe n'est pas une preuve de l'existence d'une abbaye sur l'île avant l'arrivée des Normands mais tout simplement de la longue mémoire institutionnelle des moines normands.

La vraisemblance d'une réforme monastique précoce est aussi discutable pour des raisons plus générales. Comme il a été suggéré plus haut, ce lieu sacré paraît avoir été mieux géré par des clercs qu'il ne l'aurait été par des moines, et on ne peut donc pas présumer que le clergé aurait

25. Keats-RoHAn, Katharine, " Testimonies of the Living Dead: The Martyrology-Necrology and the Necrology in the Chapter-Book of Mont-Saint-Michel (Avranches, Bibliothèque municipale, MS 214) ", dans Rollason, David et al. (éd.), The Durham " Liber Vitae " and its Context, Woodbridge, Boydell Press, 2004, p. 168-172.

26. LAPORTE, Jean, " Les Obituaires du Mont Saint-Michel ", dans LAPORTE, Jean (dir.), Millénaire Monastique, t. 1, Paris, Lethielleux, 1966, p. 725-741; KEATs-RoHAN, Katharine, "L'histoire secrète d'un sanctuaire célèbre : la réforme du Mont-Saint-Michel d'après l'analyse de son cartulaire et de ses nécrologes ", dans BouET, Pierre, OTRANTO, Giorgio et VAuchEZ, André (éd.), Culte et Pèlerinages à saint Michel en Occident, Rome, École Française de Rome, 2003, p. 152-157.

27. En détail l'analyse se décompose ainsi : 798-899 : 7 noms ; 900-999 : 3; 1000-1099 : 52 (dont 1000-1049: 28); 1100-1199: 63.

28. Einsiedeln, dont l'église n'a été consacrée qu'en 948, a produit le premier nécrologe de ce type mentionné par Joachim Wollasch : WoLLASCH, Joachim, "Les moines et la mémoire des morts ", dans IOGNA-PRAT, Dominique et PICARD, Jean-Charles (éd.), Religion et culture autour de l'an Mil, Paris, Picard, 1990, p. 47-54, particulièrement p. 49. Voir aussi IOGNA-Prat, Dominique, "Les morts dans la comptabilité céleste des Clunisiens de l'an Mil », Ibidem, p. 55- 69. 
favorisé une telle réforme, au contraire. Parce que le pouvoir concentré dans la personne d'un abbé bénédictin encourageait immanquablement les monastères à rechercher l'indépendance par rapport à ses fondateurs, il est $a$ priori peu probable qu'un évêque d'Avranches y ait été également favorable. Enfin, la datation presque certaine de la Revelatio en 851-867 et le témoignage d'Odon de Glanfeuil vont aussi à l'encontre d'une réforme bénédictine précoce sur l'île. De même, ils vont à l'encontre d'une autre thèse, approuvée d'ailleurs par Katharine Keats-Rohan, à savoir que le Mont-Tombe aurait connu une longue histoire comme monastère pratiquant " une forme bien établie de monachisme breton ", non bénédictine, que cette auteure n'a pas explicitée ${ }^{29}$.

Malgré ces objections, selon une opinion assez répandue de nos jours, le Mont-Tombe aurait connu une histoire monastique dès le $\mathrm{IX}^{\mathrm{e}}$ siècle. En dernière analyse, pour la majorité de ses défenseurs, cette conviction ne se fonde pas sur les nécrologes mais plutôt sur le texte incomplet qui conclut le journal de voyage du moine franc Bernard, en visite au Mont-Tombe vers 870 , alors qu'il achevait la dernière étape d'un pèlerinage de trois ans à Rome et en Terre Sainte. Le journal de Bernard est écrit dans un latin familier, non littéraire, et en ce qui concerne le Mont-Tombe, la chose qui l'intéressa le plus fut le prodige qui se produisait une fois par an, à la Saint-Michel, lorsque la mer à marée haute se séparait et se dressait de chaque côté, comme deux murailles, afin de permettre aux fidèles, à toute heure de la journée, de passer à pied sec du continent au sanctuaire. En aparté, il ajoute : Ibi est abbas Phinimontius Brito... ("L'abbé s'appelle ici Phinimontius, [un Breton]") ${ }^{30}$.

De ces mots, Katharine Keats-Rohan déduit qu'avant 870 la communauté insulaire était devenue bénédictine et bretonne ${ }^{31}$. En théorie, il est possible qu'une réforme monastique y ait été imposée par les Bretons, les nouveaux maîtres de l'île, juste après qu'ils eurent gagné l'Avranchin en 867. Mais cela dépasse de beaucoup le témoignage de Bernard. En premier lieu, il ne fait aucune référence à des moines. Ensuite, les communautés ecclésiastiques ont toujours accueilli des hommes de diverses origines : qu'une communauté à la frontière entre la Bretagne et la Neustrie ait choisi un chef breton à un certain moment ne nous dit pas qu'elle avait des allégeances institutionnelles envers la Bretagne.

Plus important, le témoignage de Bernard ne coïncide pas avec ce qu'on connaît de l'histoire ultérieure du Mont-Saint-Michel. Si on suppose que des bénédictins y étaient installés vers la fin des années 860, comment expli-

29. Keats-Rohan, Katharine, "Une charte de l'abbé Mayeul de Cluny et la réforme du Mont-Saint-Michel ", dans BeaurePaire, François de et Chaline, Jean-Pierre (éd.), $L a$ Normandie vers l'An Mil, Rouen, Société de l'Histoire de Normandie, 2000, p. 163.

30. Itinerarium Bernardi monachi Franci, dont quelques extraits sont publiés à nouveau avec des observations dans Bouet, Pierre et DesBordes, Olivier, Chroniques latines..., op. cit., p. 371-374.

31. KEATS-ROHAN, Katharine, "L'histoire secrète... ", art. cit., p. 143-144. 
quer que des clercs étaient toujours en possession du sanctuaire quand les Normands arrivèrent sur l'île? Même ceux qui ont des doutes sur la fiabilité de l'Introductio ne doutent pas de cette partie de l'histoire qui y est racontée : d'une part, parce qu'il est invraisemblable que les Normands aient inventé les chanoines qu'ils ont prétendu avoir chassés du Mont, et d'autre part, comme nous le verrons, parce que, selon une opinion courante, les chanoines sont en fait restés sur l'île après que les Normands se soient emparés du sanctuaire. Que serait-il arrivé entre-temps aux moines non mentionnés par Bernard?

Katharine Keats-Rohan a suggéré qu'il y avait peut-être eu une scission dans la communauté au moment de sa "réforme " et qu'ensuite deux communautés, l'une de clercs, l'autre de moines, auraient coexisté sur le Mont ${ }^{32}$. Mais, en ce cas, les moines bénédictins du Ix ${ }^{\mathrm{e}}$ siècle auraient dû céder le sanctuaire, le rendant encore une fois au clergé, sinon les Normands n'y auraient pas trouvé des clercs en place. Comment expliquer cette deuxième réforme et pourquoi n'y avait-il aucun signe au Mont de la présence de ces bénédictins au moment où les Normands en ont installé d'autres, venus de Saint-Wandrille et d'ailleurs, dans les années 960 ? L'existence, une centaine d'années plus tôt, d'une communauté de moines, suggérée par l'usage du mot abbas dans le journal de Bernard, dépend trop d'un enchevêtrement d'invraisemblances pour être crédible.

D'autant qu'il y a d'autres possibilités pour expliquer ce mot. Le terme abbas n'a pas toujours signifié le chef d'un monastère. En Gaule mérovingienne, il était aussi employé pour le prêtre ayant en charge une église célèbre par des tombes ou des reliques et pourvue de plusieurs prêtres subordonnés $^{33}$. Cela ressemble à la situation au Mont-Tombe. D'ailleurs, à la fin du $\mathrm{x}^{\mathrm{e}}$ siècle, nous connaissons l'existence de quatre ou cinq églises ou oratoires sur l'île, et il pouvait bien en exister d'autres ${ }^{34}$. Ceci suggère un ensemble de clercs qui parfois agissaient individuellement. Pour décrire le dirigeant de cette structure insolite, Bernard a peut-être employé une désignation tirée du vocabulaire monastique connue de lui car, tout simplement, il ne connaissait aucun autre terme convenable; ou bien peut-être s'est-il trompé à propos de la structure formelle de la communauté? Quelle que soit l'explication, son abbas n'est pas une preuve de l'existence d'un monastère sur l'île vers 870 .

En ce qui concerne le Mont-Saint-Michel, il ne nous reste donc aucune narration pré-normande plausible, à part l'histoire traditionnelle selon laquelle le sanctuaire était toujours desservi par la communauté aubertienne au milieu du $\mathrm{X}^{\mathrm{e}}$ siècle. C'est peut-être pourquoi quelques historiens qui veulent bouleverser l'interprétation traditionnelle s'acharnent à dis-

32. Ibidem.

33. GodDING, Robert, Prêtres en Gaule mérovingienne, Bruxelles, Société des Bollandistes, 2001, p. 229-239.

34. GANDY, George, « Who built what...? », art. cit., p. 166-167. 
créditer toute preuve soutenant l'affirmation normande, selon laquelle les Normands avaient fondé l'abbaye en 965 . Trois sources sont en question.

\section{Le diplôme de Lothaire, roi des Francs de l'Ouest, daté du 7 février 966}

Un diplôme prétendument délivré par Lothaire en 966 et connu seulement de deux pseudo-originaux plus tardifs raconte qu'au lieu appelé le Mont-Saint-Michel, situé au milieu de la mer, Richard marquis des Normands, a récemment rassemblé une communauté de moines avec le consentement du pape Jean. L'acte déclare qu'à la requête du pape, du marquis et d'Hugues archevêque de Rouen, à la province duquel le monastère appartenait, le roi confirme que les moines peuvent y rester pour toujours. Les éditeurs des actes de Lothaire, Louis Halphen et Ferdinand Lot, ont considéré ce diplôme comme authentique à l'exception de l'interpolation de la bulle de Jean XIII qu'ils ont, et quasiment tous les spécialistes après eux, considérée comme fausse et datée du $\mathrm{XI}^{\mathrm{e}}$ siècle $^{35}$.

Cependant, depuis quelques décennies, la thèse d'une falsification totale est acceptée en raison de sa réitération fréquente, au point que l'inauthenticité supposée de ce diplôme est utilisée pour soutenir des affirmations n'ayant absolument rien à voir avec la question. Pour Mathieu Arnoux, par exemple, sa falsification (faite à Rouen, pense-t-il) serait une preuve qu'à la fin du $x^{e}$ siècle l'archiépiscopat de Rouen aurait voulu annexer le diocèse d'Avranches, une proposition qui ne repose sur rien si le diplôme est en réalité authentique ${ }^{36}$.

Selon Katharine Keats-Rohan, le diplôme est bien évidemment une falsification du XI ${ }^{\mathrm{e}}$ siècle parce qu'une demande d'un diplôme royal visant à la restauration ou à la réforme d'une abbaye aurait nécessairement dû venir du dux francorum, Hugues Capet, en tant que senior de Richard I ${ }^{\text {er }}$. Il aurait aussi fallu que ce dernier nomme l'abbé du monastère et confirme sa dotation foncière ${ }^{37}$. Rien de tout cela ne s'applique ici. En effet, des écarts à la norme en termes de procédures de fondation ne peuvent pas prouver une falsification, car dans chaque cas ces écarts peuvent être expliqués autrement : par exemple, Hugues Capet a peut-être désapprouvé les circonstances dans lesquelles son beau-frère Richard I ${ }^{\mathrm{er}}$ avait établi les bénédictins sur l'île; l'abbé y avait peut-être participé malgré lui ; à ce moment-là les moines n'avaient sans doute aucun titre clair de possession d'aucune des terres... Comme nous le verrons, tous ces éléments pouvaient compliquer les choses.

Le plus important reste que le diplôme du Mont-Saint-Michel, comme Pierre Bouet l'a récemment montré, suit correctement toutes les formules

35. Recueil..., HALPHEN, Louis et Lot, Ferdinand (éd.), op. cit., p. 53-57.

36. ARnoux, Mathieu, "Before the "Gesta Normannorum" and beyond Dudo : some evidence on early Norman Historiography ", Anglo-Norman Studies, 22, 2000, p. 37-38.

37. Keats-Rohan, Katharine, Cartulary..., op. cit., p. 19-20. 
employées par Gezo qui a rédigé les actes de Lothaire entre 958 et 969, et que les formules de Gezo diffèrent de celles de son prédécesseur Gui et de celles de son successeur Adalbero ${ }^{38}$. Il est inconcevable que des moines du Mont aient connu assez précisément les pratiques de la chancellerie royale, vers la fin du $\mathrm{x}^{\mathrm{e}}$ ou au début du XI ${ }^{\mathrm{e}}$ siècle, pour être en mesure de contrefaire un diplôme de façon si convaincante. Ils n'auraient pas pu non plus trouver aisément un modèle à copier. Comme Katharine Keats-Rohan elle-même l'a fait remarquer, Lothaire n'a en effet délivré aucun autre diplôme aux abbayes du nord-ouest de la France ${ }^{39}$. Il est donc évident qu'une fois écartée l'interpolation concernant la bulle de Jean XIII, le diplôme est authentique; et nous pouvons noter qu'en décrivant l'installation des bénédictins au Mont comme récente, il est compatible avec les annales montoises qui imputent l'événement à $965^{40}$.

\section{L'Inventio et miracula sancti Vulfranni (vers 1053-1054)}

La tradition selon laquelle Richard ${ }^{\mathrm{er}}$ aurait fait venir Mainard de SaintWandrille pour être le premier abbé du Mont-Saint-Michel ne tient pas de la fantaisie d'un scribe montois faisant tout son possible pour établir des origines non bretonnes à son abbaye. Du moins, nous la trouvons d'abord dans le texte de fondation de Saint-Wandrille elle-même, l'Inventio et miracula sancti Vulfranni ${ }^{41}$. Saint-Wandrille, refondation de l'ancienne abbaye de Fontenelle, proche de Rouen, avait été restaurée en 960 par des moines venus de Gand sous l'égide d'un certain Mainard, disciple du réformateur flamand Gérard de Brogne. Par la suite, on sait que pendant plusieurs décennies Saint-Wandrille eut bien du mal à subsister ${ }^{42}$, et le départ forcé de son chef pour le Mont-Saint-Michel, sans doute avec plusieurs de ses moines, dans la phase initiale du projet, apparaît comme une cause extrêmement plausible de telles difficultés. Vers 965, Jumièges, Saint-Ouen peutêtre, et la toute nouvelle Saint-Wandrille étaient les seuls monastères de Normandie d'où la première cohorte des bénédictins du Mont aurait pu être extraite. Il est certain, en outre, que l'abbé du Mont entre 966 et 991 était un moine nommé Mainard ${ }^{43}$. Ainsi, à première vue, le compte rendu de l'Inventio semble tout à fait crédible.

38. Par exemple, pour la suscription Gezo a employé la formule Lotharius gratia Dei rex, comme ici, ou divina dispensante clementia rex, alors que Gui et Adalbéron ont employé presque toujours Francorum avec rex et ont préféré divina propitiante clementia Francorum rex (Gui) ou misericordia Dei Francorum rex (Adalbero) : BouET, Pierre et DESBORDEs, Olivier, Chroniques latines..., op. cit., p. 158.

39. Keats-RoHan, Katharine, " L'histoire secrète... ", art. cit., p. 145.

40. Keats-Rohan, Katharine, Cartulary..., op. cit., p. 187.

41. Inventio et miracula sancti Vulfranni, LAPORTE, Jean (éd.), Rouen, Société de l'Histoire de Normandie, 1938.

42. GAZEAU, Véronique, Normannia monastica. Les princes normands et les abbés bénédictins ( $X^{e}$-XII siècle), Caen, CRAHM, 2007, t. 1, p. 202-204.

43. LAPORTE, Jean, "Les séries abbatiale et priorale du Mont Saint-Michel ", dans LAPORTE, Jean (dir.), Millénaire Monastique, t. 1, Paris, Lethielleux, 1966, p. 270. 
Katharine Keats-Rohan a cependant avancé plusieurs arguments mettant en doute l'histoire racontée à Saint-Wandrille. Tout d'abord, les sources montoises précoces - elle mentionne explicitement les écrits historiques de l'abbé Robert de Torigni (1154-1186) et les premières annales de l'abbaye, datées d'après 1030 - ne mentionnent pas d'où est venu leur premier abbé Mainard ${ }^{44}$. Cela n'a cependant rien d'étonnant. Les premières annales du Mont se composent de notes succinctes sur un petit nombre d'événements et il ne faut pas s'attendre à ce qu'elles discourent longuement sur les carrières antérieures des abbés. Robert de Torigni donne parfois de telles informations pour les abbés qui ont dirigé différentes abbayes normandes, mais d'autres fois il n'en donne pas. On ne peut tirer aucune conclusion spécifique de ce manque de cohérence ${ }^{45}$.

Katharine Keats-Rohan pense par ailleurs que le récit du départ précoce de Mainard pour le Mont que l'on trouve dans l'Inventio a peut-être à l'origine servi à dissimuler une hypothétique médiocrité de Mainard à la tête de Saint-Wandrille pendant quelques décennies. Elle suggère que le "mythe " de ce départ aurait peut-être été conçu par l'éminent abbé de Saint-Wandrille, Gérard, nommé en 1008 par Richard II pour restaurer la réputation de l'abbaye, et elle fait également la suggestion, peu plausible, comme l'a démontré Véronique Gazeau, que ce Gérard aurait d'abord été un moine du Mont-Saint-Michel et qu'il aurait ainsi été informé des dates de l'abbatiat de "l'autre " abbé Mainard, celui du Mont ${ }^{46}$. Mais le nombre d'exigences non satisfaites par une telle explication met plutôt l'accent sur la crédibilité de l'histoire originale. En outre, le nécrologe du XI ${ }^{\mathrm{e}}$ siècle atteste qu'au Mont-Saint-Michel des prières étaient effectuées en faveur des moines de Saint-Bavon et de Saint-Pierre-au-Mont-Blandin, deux abbayes de Gand réformées par Gérard de Brogne, ainsi qu'en faveur de la comtesse Alfrida, la mère d'Arnoul I ${ }^{\mathrm{er}}$ de Flandres, patron de de Brogne. Les moines de Saint-Wandrille figuraient aussi plus souvent dans leurs prières que ceux de toute autre abbaye normande ${ }^{47}$. Les liens entre le Mont-Saint-Michel et Saint-Wandrille apparaissent donc assez clairs. L'explication préférée par Katharine Keats-Rohan, selon laquelle le Mont aurait entretenu des liens particuliers avec la Flandre grâce à des prétendues relations avec l'Angleterre dépend encore une fois d'hypothèses complexes non corroborées

44. KEATS-Rohan, Katharine, " L'histoire secrète... ", art. cit., p. 151-152.

45. Véronique Gazeau, dans un résumé du débat à propos de l'histoire de Mainard selon l'Inventio, pour la plupart excellent, a peut-être elle-même émis un doute qui, de façon similaire, manque de substance. Elle accorde en effet du crédit aux différences liturgiques entre Saint-Wandrille et le Mont-Saint-Michel qui ressortent de leurs sacramentaires respectifs de la fin du XI ${ }^{\mathrm{e}}$ siècle, différences qui l'incitent à penser que le lien proposé dans l'Inventio est plutôt douteux. Cependant, les interventions liturgiques de plusieurs générations d'abbés et de chantres de chacun des deux monastères auraient dû rendre ces divergences prévisibles. On ne sait rien de leurs liturgies au milieu du X ${ }^{\mathrm{e}}$ siècle. Cf. GAZEAU, Véronique, Normannia monastica..., op. cit., t. 1, p. 205-211.

46. KeAts-RoHAn, Katharine, "L'histoire secrète... ", art. cit., p. 156-157. Cf. GAZEAU, Véronique, Normannia monastica..., op. cit., t. 1, p. 207-208.

47. Bouet, Pierre et Desbordes, Olivier, Chroniques latines..., op. cit., p. 168-172. 
par la documentation. Elle apparaît ainsi beaucoup moins convaincante que l'histoire racontée au XII ${ }^{\mathrm{e}}$ siècle.

Katharine Keats-Rohan a enfin avancé que le Mainard du Mont n'était pas flamand mais " manifestement un noble neustrien avec de très bonnes relations ", qui était parent, suggère-t-elle, des comtes et vicomtes du Mans. Bien avant l'arrivée au Mont des Normands en 965, ce Mainard «était abbé [du Mont] depuis longtemps sans doute, peut-être dès les années 940 ou $950^{48}$ ». Mais ce n'est qu'une pure spéculation. Elle n'a pu identifier aucun Mainard issu de ce groupe généalogique ayant la durée de vie requise et Véronique Gazeau, elle-même également spécialiste de prosopographie, considère cette hypothèse comme " fragile ${ }^{49}$ ". Katharine Keats-Rohan n'a également aucune preuve que le Mainard du Mont y ait rempli la fonction d'abbé avant l'arrivée des Normands.

À tout point de vue, que le Mainard de Saint-Wandrille soit aussi celui du Mont reste l'hypothèse la plus convaincante. Elle correspond aux implications du diplôme confirmant que Richard I ${ }^{\mathrm{er}}$ est intervenu au Mont-SaintMichel au milieu des années 960 . Elle corrobore aussi l'idée que l'établissement de la nouvelle abbaye s'est fait sans véritable planification.

\section{La charte de Maïeul de Cluny (966?)}

Par une charte copiée dans le cartulaire du Mont-Saint-Michel, un petit champ de vigne situé à Marmoutier, sur la Loire, fut concédé à l'abbaye par l'abbé Maïeul de Cluny avec l'accord des fratres de Cluny et de Marmoutier ${ }^{50}$. La copie du cartulaire est datée regnante Lothario rege anno xli, mais comme Lothaire (954-986) est décédé dans la trente-deuxième année de son règne, il est évident qu'il s'agit d'une erreur. Dans son édition du cartulaire, Katharine Keats-Rohan remarque très justement : "It was clearly intended at the Mont that the date should be read as anno xii, the twelfth year, i.e. 966. The use of an ' $l$ ' form for a long ' $i$ ' occurs elsewhere in the cartulary $[\ldots]^{51}$ ".

L'authenticité de la charte n'est pas en doute ${ }^{52}$, mais sa datation est problématique puisqu'en 966 Cluny n'est pas connue pour avoir eu de lien institutionnel avec Marmoutier, bien que l'abbaye bourguignonne ait été active dans la région dès avant cette date en réformant l'abbaye de Saint-Julien qui appartenait au même cercle tourangeau ${ }^{53}$. Pendant les années 960 ,

48. Keats-Rohan, Katharine, « L'histoire secrète... ", art. cit., p. 158-159.

49. GAZEAU, Véronique, Normannia monastica..., op. cit., t. 1, p. 211.

50. Keats-Rohan, Katharine, Cartulary ..., op. cit., numéro 32, p. 114-115. Dans la charte le terrain est décrit tout simplement comme terra, mais par la suite il est certain qu'il a fourni du vin aux moines (ibidem, p. 233).

51. Keats-Rohan, Katharine, Cartulary..., op. cit., p. 114.

52. Keats-Rohan, Katharine, "Une charte... », art. cit., p. 165; et idem, Cartulary..., op. cit., p. 39.

53. FARMER, Susan, Communities of St Martin : Legend and ritual in medieval Tours, Ithaca, Cornell University Press, 1991, p. 30. 
Marmoutier était une pauvre communauté de chanoines, sous le contrôle d'Hugues Capet comme abbé laïque, ayant subi des revers de fortune au cours des $\mathrm{IX}^{\mathrm{e}}$ et $\mathrm{X}^{\mathrm{e}}$ siècles ${ }^{54}$. On sait qu'Hugues Capet était ami et un soutien de Maïeul, mais c'est en fait Eudes I $^{\mathrm{er}}$, comte de Blois, sous l'autorité duquel Capet paraît avoir placé Marmoutier, qui, vers 985-986, demanda à Maïeul de réformer la communauté, faisant appel à des moines de Cluny pour ce faire $^{55}$. Le don en faveur du Mont pourrait donc dater de cette période-là.

À l'encontre d'une datation si tardive, il faut cependant prendre en compte les circonstances suivantes : tout d'abord, en 986, Maïeul a engagé un assistant, or celui-ci n'a pas ratifié cette charte ${ }^{56}$; ensuite, un des officiers de Cluny qui, lui, était signataire de la charte, un prêtre nommé Otger, apparaît soit comme scribe, soit comme témoin, dans cinq autres chartes clunisiennes, mais seulement entre 966 et $972^{57}$; enfin, dans la charte, Maïeul déclare effectuer la donation avec l'accord de ses fratres, mais en faveur des monachi qui servent saint Michel, une distinction qui suggère que la communauté de Marmoutier se composait encore de chanoines et non de moines. Il ne faut donc pas rejeter d'emblée la possibilité que la réforme monastique d'Eudes se soit appuyée sur un rôle consultatif ou de surveillance préexistant de Cluny, peut-être exercé à titre intermittent et non documenté, alors qu'Hugues Capet était toujours abbé laïque de la maison, une position qu'il occupait depuis la mort de son père en 956 . Par conséquent le don de Maïeul en faveur du Mont peut dater de 966, soit quelques mois après l'arrivée de Mainard et ses moines au Mont.

Il convient également de réfléchir à la teneur de la charte. C'est à Marmoutier, au IV ${ }^{\mathrm{e}}$ siècle, que saint Martin de Tours, avec 84 de ses disciples, habitant des grottes et des cabanes proches du fleuve, introduisit le monachisme en Gaule ${ }^{58}$. Maïeul de Cluny, lui, était l'abbé le plus important de son époque, et son don au Mont d'une petite vigne de cette propriété était grandement significatif. Premièrement, le raisin représentait le vin de l'eucharistie et ainsi l'espoir que le salut serait offert aux moines du Mont; mais au-delà, la vigne était le symbole des relations entre Dieu et son peuple. Le vignoble était le lieu protégé par Dieu, où les vignes, ses enfants, prospéraient sous sa garde; par extension, la vigne symbolisait aussi l'Église. On peut inférer que par ce don d'un vignoble, Maïeul investissait Mainard du devoir de réimplanter le monachisme, peut-être même le christianisme, en Normandie. On oublie facilement combien le paysage ecclésiastique de la province, toujours dépourvue d'évêques et de monastères même cinquante ans après la conversion publique de Rollon au christianisme, dut paraître

54. Ibidem, p. 19 et 35.

55. Ibid., p. 67-68. Cf. KEATS-ROHAN, Katharine, "Une charte... ", art. cit., p. 165, qui date la réforme de Maïeul vers 982 mais n'en donne pas de citation.

56. LAPORTE, Jean, "L'Abbaye du Mont-Saint-Michel aux X et XI ${ }^{\mathrm{e}}$ siècles ", dans LAPORTE, Jean (dir.), Millénaire Monastique, t. 1, p. 61.

57. Keats-Rohan, Katharine, Cartulary..., op. cit., p. 232-233.

58. Dunn, Marilyn, The Emergence of Monasticism. From the Desert Fathers to the Early Middle Ages, Oxford, Blackwell Publishing, 2000, p. 62-64. 
désert à des religieux venus d'ailleurs. Les vignes provenant spécifiquement de Marmoutier étaient aussi chargées de sens parce que, pour les premiers moines de saint Martin, les falaises trouées d'habitations troglodytes avaient représenté une solitude ascétique rappelant la vie des pères du désert en Égypte, bien qu'en même temps, ce fût un endroit entouré des riches terres du Val de Loire, illustrant ainsi la munificence de la bienveillance de Dieu. En termes ecclésiastiques, la Basse-Normandie du milieu du $\mathrm{X}^{\mathrm{e}}$ siècle était aussi un désert, mais un désert auquel les vignes de Marmoutier promettaient qu'une métamorphose était du domaine du possible.

On ne peut pas être certain que le don de Maïeul au Mont-Saint-Michel date de 966, et pas des années 980, mais son sens hautement symbolique confirme que le moment le plus opportun et le plus propice aurait été cette année, juste après l'arrivée des bénédictins sur l'île.

Nous avons donc de manière certaine deux, et peut-être même trois, raisons d'avoir confiance en l'affirmation des Normands selon laquelle ils avaient introduit les bénédictins au Mont-Saint-Michel et que cela s'était produit en 965 , ainsi qu'il est mentionné dans les premières annales de l'abbaye.

\section{L'installation des moines}

De toute évidence, Richard $\mathrm{I}^{\mathrm{er}}$ avait envers l'Église une attitude plus ambivalente que son père Guillaume Longue Épée. Richard ne correspondait pas au modèle du chrétien pieux selon les conventions, peut-être à cause de son éducation à Bayeux parmi des Scandinaves connus pour leur pragmatisme en matière religieuse ${ }^{59}$. $\mathrm{Si}$, à la fin du siècle, les Normands étaient toujours réputés irrémédiablement païens selon les ecclésiastiques orthodoxes comme Guillaume de Volpiano, c'était sans doute dû à la perception selon laquelle Richard n'avait pas soutenu les intérêts de l'Église avec le zèle attendu d'un prince chrétien et qu'il avait peut-être, en outre, conservé des croyances hétérodoxes.

Au cours d'un règne de plus de 50 ans, Richard ne fit rien pour reconstruire l'Église en tant qu'institution avant l'approche de sa mort. Le siège métropolitain mis à part, aucun des sept diocèses de la province ne fut pourvu en permanence avant les années $986-990^{60}$. François Neveux a suggéré que Richard n'établit une autorité adéquate sur son aristocratie que tard dans sa vie, pour pouvoir recouvrer d'elle les propriétés ecclésiastiques qu'elle avait détournées ${ }^{61}$. Mais, si l'on écarte l'idée selon laquelle les

59. Dudon de Saint-Quentin, De moribus..., op. cit., p. 221-2. Van ToRHoudt, Éric, Centralité et marginalité..., op. cit., p. 168-171.

60. NEveux, François, La Normandie des ducs, p. 56-57 et 274-276. Au cours du Xe siècle, des évêques furent nommés sur le siège de Coutances, mais ils résidaient à Rouen, et le firent encore pendant une bonne partie du siècle suivant. À l'évidence ils n'ont pas concrètement pris en charge leur diocèse.

61. Ibidem, p. 274-276. 
premiers ducs normands n'auraient pas vraiment désapprouvé les droits exercés par des laïcs sur les biens de l'Église ${ }^{62}$, cette analyse n'explique pas qu'en définitive Richard se soit soudain décidé à modifier les choses à travers le duché en l'espace de deux à quatre ans ${ }^{63}$. Comme cela dut paraître évident à Guillaume de Volpiano, ces actions trahissaient manifestement une mentalité instrumentaliste : Richard a pourvu les évêchés, quand il l'a fait, non à cause de la disparition soudaine d'une contrainte puissante et omniprésente qui aurait permis au rayonnement de sa piété de se manifester, mais parce qu'il a vu qu'il était de son intérêt de le faire (et donc il aurait pu le faire plus tôt) ${ }^{64}$.

Le traitement accordé aux moines sous Richard I ${ }^{\mathrm{er}}$ a suivi une trajectoire parallèle. À l'exception de deux cas, les abbayes de Fontenelle/SaintWandrille et du Mont-Saint-Michel, le duc ne promut ni ne facilita le monachisme avant une période tardive de sa vie, vers l'an $990^{65}$. Au milieu du $\mathrm{IX}^{\mathrm{e}}$ siècle, les moines de Fontanelle avaient fui les Vikings, en s'installant finalement à l'abbaye de Saint-Pierre-au-Mont-Blandin près de Gand. Vers 944-953, l'abbé réformateur de Saint-Pierre, Gérard de Brogne, ramena un corps de moines en Normandie, mais Richard refusa de leur restituer les propriétés d'origine et la refondation ne réussit pas. En 960, Gérard de Brogne étant décédé l'année précédente, il y eut une deuxième tentative sous son disciple Mainard. Cette fois, le duc permit la refondation; mais ses anciennes terres ne furent rendues à l'abbaye que plusieurs décennies plus tard et ce fut donc d'abord une simple dépendance de Saint-Pierre de Gand, sans abbé à strictement parler ${ }^{66}$.

62. VAn Torhoud, Éric, Centralité et marginalité..., op. cit., p. 392.

63. Selon une révision récente des dates probables de nomination des évêques, au moins trois des cinq sièges pourvus à cette époque-là suite à une longue vacance, et peut-être tous les cinq, ont été attribués vers 989-90, c'est-à-dire seulement en l'espace de deux ans (ALLEN, Richard, The Norman Episcopate, 989-1110, thèse de doctorat, University of Glasgow, 2009, fig. 3, p. 14).

64. Les raisons qui expliquent son changement de conduite dépassent les limites de cet article, mais il paraît s'être lancé dans une stratégie, poursuivie par ses héritiers, d'intégration du duché dans les dynasties européennes royales et impériales, ce qui l'entraîna en partie à définir un lignage d'héritiers légitimes (d'où la christianisation des mariages ducaux) et la restauration de la hiérarchie ecclésiastique en Normandie. Il a été suggéré, d'une manière plausible, que les unions informelles des Rollonides avec des femmes de l'entourage ducal, scandinaves, franques ou bretonnes, particulièrement caractéristiques du x ${ }^{\mathrm{e}}$ siècle, avaient encouragé l'aristocratie normande à s'unir autour de la lignée ducale, et que, par conséquent à partir des années 990, ce degré de sécurité interne atteint, il lui est devenu possible de mener une stratégie matrimoniale plus exogame (VAN TORHOUDT, Éric, Centralité et marginalité..., op. cit., p. 606-637, en particulier p. 633-634).

65. Selon une pancarte du XII ${ }^{\mathrm{e}}$ siècle, Richard I Ier refonda aussi Saint-Taurin d'Évreux après avoir repris la ville éponyme à Thibaud de Blois en 962, mais la première mention de l'abbaye ne date que de 989. La sincérité de cette tradition est donc incertaine, et même douteuse : GAZEAU, Véronique, Normannia monastica..., op. cit., t. 1, p. 9; et VAN TORHOUDT, Éric, Centralité et marginalité..., op. cit., p. 187-190.

66. GAzEAU, Véronique, Normannnia monastica..., op. cit., t. 1, p. 202-203. 
Richard n'était pas l'instigateur de ce projet et il fit très peu pour le faciliter. Très tôt effectivement, comme nous l'avons vu, il mit presque fin à l'entreprise en faisant appel à Mainard pour la fondation du Mont-SaintMichel. Néanmoins, on peut expliquer cette intervention, peu profitable à Saint-Wandrille, si l'on considère l'installation des bénédictins au Mont surtout comme un geste politique sommairement organisé. Celui-ci se produisit en conclusion d'une guerre qui opposait le duc au roi franc Lothaire, à l'empereur allemand Otton le Grand et à Thibaud, comte de Blois et de Chartres, "bête noire » des Normands ${ }^{67}$. Une paix signée à Gisors en 966 mit fin aux hostilités et par la suite la Normandie ne subit plus aucune autre invasion au cours des trente dernières années de la vie du duc ${ }^{68}$. Il est très probable que la fondation de l'abbaye du Mont-Saint-Michel constitua un élément du processus de paix. Les deux choses sont liées par le diplôme que Lothaire délivra en février 966, confirmant formellement que les moines récemment installés sur l'île par Richard avaient le droit inviolable d'y rester pour toujours.

Le diplôme paraît attester de négociations conduites au niveau de la cour et du haut clergé de la province, et apparaît comme un élément du système de don et contre-don, caractéristique du processus médiéval de réconciliation. On peut présumer que Lothaire a partagé avec la plupart de ses contemporains le soupçon que les Normands étaient toujours païens et qu'il a accueilli l'acte de fondation d'une abbaye par Richard comme un témoignage de son désir d'être reconnu à l'avenir comme un souverain vraiment chrétien, malgré le caractère manifestement politique de son acte (souligné par le fait que le duc n'a pas, par la suite, conféré de biens à l'abbaye ni fondé d'autres monastères avant 990).

Richard a sans doute apprécié que l'îlot soit déjà pourvu de bâtiments appropriés, que les locaux soient occupés par une communauté d'un genre obscur, assurément non bénédictine, qu'en l'absence d'un évêque d'Avranches qui aurait pu protester (Richard n'en nommant un que vers 990), il pouvait discrètement remplacer par des moines. Sans frais pour le duc, la maison était prête pour l'emménagement.

D'un autre côté, le diplôme, c'est-à-dire le contre-don de Lothaire, n'était pas seulement une reconnaissance de l'acte de fondation de l'abbaye par Richard. L'important tenait au fait qu'il concédait à un ordre de moines le droit de demeurer sur l'île à perpétuité. Quand, quelques années plus tôt, Gérard de Brogne, le mentor de Mainard, qui réformait alors l'abbaye de Saint-Pierre-au-Mont-Blandin, avait cherché à obtenir un acte semblable du père de Lothaire, Louis d'Outremer, son but avait été de faire confirmer les droits de la nouvelle communauté sur la propriété appelée à être prise en charge ${ }^{69}$. En revanche, le diplôme de Lothaire, en se concentrant sur le droit

67. NeveuX, François, Normandie des ducs..., op. cit., p. 50-51.

68. Sur la datation de Gisors, voir Pоттs, Cassandra, Monastic Revival and Regional Identity in Early Normandy, Woodbridge, Boydell Press, 1997, p. 81-82, note 3.

69. LAPORTE, Jean, " L'Abbaye du Mont Saint-Michel aux X et XI siècles ", dans LAPORTE, Jean (dir.), Millénaire Monastique, t. 1, p. 61. 
d'occupation de l'île par les moines, suggère, en ce qui concerne le Mont, que cette question était vraiment délicate et qu'il s'agissait de l'aspect de la fondation le plus manifestement sujet à contestation. Autrement dit, c'était parce que le diplôme royal sanctionnait la mise à l'écart des premiers occupants du Mont que Richard en avait tant besoin. Implicitement, le diplôme approuvait une expropriation arbitraire et il se peut qu'Hugues Capet l'ait désapprouvé pour cette raison; de même, il mettait très probablement mal à l'aise le nouvel abbé du Mont. Ni l'un, ni l'autre n'étaient associés à l'acte ${ }^{70}$. Quant aux droits des moines à prendre possession du patrimoine de leurs prédécesseurs, le diplôme n'en dit rien, sans doute parce que la prise de contrôle non consensuelle du sanctuaire en faisait un objet de litige, non seulement avec les clercs expropriés mais aussi avec les familles des anciens bienfaiteurs de la communauté. Lothaire et le duc ont sans doute préféré laisser les moines régler la question du mieux qu'ils pouvaient.

Considérer l'installation des moines dans son contexte politique nous aide à comprendre les raisons pour lesquelles Richard a agi ainsi précisément en 965-966 et pourquoi il a négligé sa fondation par la suite. L'authenticité du diplôme de Lothaire est aussi soulignée par sa pertinence fonctionnelle en termes d'établissement des nouveaux habitants sur l'île.

Une fois installés et reconnus par le roi, les bénédictins, ayant servi le but immédiat de Richard, furent ignorés du duc et de sa cour pendant plusieurs décennies, au point que quelques historiens ont douté qu'aucun événement significatif du point de vue institutionnel ait pu se produire au Mont pendant les années 960. En conséquence, les premières années de l'abbaye furent loin d'être prospères. Le don très symbolique de Maïeul mis à part, les moines n'ont reçu aucune donation foncière pendant les vingtcinq premières années de leur présence sur le Mont. Peut-être les donateurs potentiels ont-ils hésité jusqu'à ce qu'il devienne évident que l'abbaye soit acceptée comme héritière des propriétés qui avaient autrefois appartenu à la communauté de clercs. Finalement ce degré de légitimité fut atteint. Vers l'an mil, par exemple, Ivo fils de Fulcoin, "restituait " à l'abbaye huit villae que ses ancêtres avaient cédées aux prédécesseurs des moines mais que ceux-ci avaient perdues pendant l'époque viking ${ }^{71}$.

Plusieurs signes suggèrent qu'un quart de siècle passa avant que l'abbaye ne commence à prospérer. À partir de l'an 990 environ, on constate cependant un afflux de nouvelles donations, - quoiqu'elles ne proviennent pas de la famille ducale ni de l'aristocratie normande, mais plutôt de Bretagne et du Maine ${ }^{72}$-, et simultanément, d'ambitieux travaux de construction, l'essor de la production manuscrite de ce qui serait

70. Voir ci-dessus, p. 17.

71. Keats-RoHAn, Katharine, Cartulary..., op. cit., p. 108-110. Vraisemblablement, Fulcoin aurait restitué ces propriétés au clergé si les clercs habitaient encore sur l'île : quant à ce débat, voir plus loin.

72. PotTs, Cassandra, Monastic Revival..., op. cit., p. 87-96. 
un des plus importants scriptoria du nord-ouest de la France, enfin une augmentation considérable du nombre des moines ${ }^{73}$. Sans qu'on puisse l'expliquer, une liste de noms de 50 moines du Mont toujours vivants et de 40 moines décédés fut ajoutée, entre 1005 et 1009, à un sacramentaire destiné à Fleury. À l'évidence, le nombre des morts par rapport aux vivants a été sous-estimé ${ }^{74}$. Néanmoins, 40 morts serait un nombre incroyablement faible si, comme l'a avancé Katharine Keats-Rohan, l'histoire de l'abbaye remontait au IX ${ }^{\mathrm{e}}$ siècle. Le chiffre des moines décédés serait également beaucoup trop faible pour un monastère fondé une quarantaine d'années auparavant si celui-ci avait régulièrement abrité 50 moines. Ceci suggère très probablement qu'une population d'abord peu nombreuse avait subi une augmentation importante pendant la dizaine ou quinzaine d'années précédant immédiatement le recensement, c'est-à-dire dans les années 990.

Durant ses vieux jours Richard $\mathrm{I}^{\mathrm{er}}$ commença à soutenir le développement du monachisme en Normandie, de même qu'il pourvut les sièges des évêchés qu'il avait laissés vacants auparavant. Au Mont-Saint-Michel, il paraît avoir financé la restauration du toit de l'église et des bâtiments monastiques sérieusement endommagés à la suite d'un incendie vers $992^{75}$, peut-être en conformité avec sa nouvelle politique envers l'Église en général, ou bien pour contrer l'influence de la Bretagne et du Maine, d'où les dons avaient récemment commencé à affluer en faveur des moines montois. Toutefois, bien que la générosité de ces régions envers l'abbaye ait continué pendant les années 990-1010 environ, il n'y eut aucune d'autre donation de la part de la famille ducale pendant cette période.

Une autre explication possible mérite réflexion, même si elle est moins généreuse envers les motivations du duc. Chose curieuse en effet, au sudest de l'église abbatiale (aujourd'hui Notre-Dame-sous-Terre), subsistent des vestiges d'un bâtiment de forme basilicale qui paraît dater de la fin du $\mathrm{X}^{\mathrm{e}}$ ou du début du $\mathrm{XI}^{\mathrm{e}}$ siècle et qui était d'une qualité et d'une ampleur surprenantes. Sa destination est inconnue mais il y a plusieurs raisons de

73. En ce qui concerne les dons fonciers, voir numéros 22, 26-28 and 47, et aussi Appendix ii.1, parmi les chartes énumérées dans KeATs-RoHAN, Katharine, Cartulary..., op. cit., p. 55-62. Sur l'histoire précoce du scriptorium, voir ALEXANDER, Jonathan, Norman Illustration at Mont Saint Michel, 966-1100, Oxford, Clarendon Press, 1970, p. 23-126 et DosDat, Monique, L'Enluminure romane au Mont-Saint-Michel, $X^{e}$-XII ${ }^{e}$ siècle, Rennes, OuestFrance, 2006, p. 25-67.

74. Quelques-uns ont peut-être été oubliés, d'autres sont décédés après avoir changé de monastère. Il est probable que la raison principale de la sous-estimation des morts ait été la proportion inconnue de moines décédés après avoir définitivement abandonné la vie religieuse. Le contenu de la liste est reproduit dans BouET, Pierre et DESBORDEs, Olivier, Chroniques latines..., op. cit., p. 379-380. La provenance et la datation du recensement, mais pas ses implications démographiques, sont discutées en détail dans GRÉMONT, Denis et DonnAT, Lin, " Fleury, le Mont Saint-Michel et l'Angleterre à la fin du x $x^{\mathrm{e}}$ siècle et au début du XI ${ }^{\mathrm{e}}$ siècle ", dans LAPORTE, Jean (dir.), Millénaire Monastique..., op. cit., t. 1, p. 751-793.

75. Dudon nous dit que le duc y a construit une église, mais l'abbatiale n'était pas détruite par le feu et il est plus probable qu'il a contribué à reconstruire son toit : GANDY, George, "Who built what...? ", op. cit., p. 165-166. 
penser que ce n'était pas une église, mais un édifice construit pour un usage séculier. Il est possible qu'il ait été imposé aux moines par Richard I ${ }^{\text {er }}$ en prévision des noces de ses enfants, Richard et Havoise, avec les enfants du duc Conan de Bretagne, Judith et Geoffroi, noces qui furent célébrées au Mont peu après l'an mil ${ }^{76}$. Quoi qu'il en soit, si le duc en a vraiment été responsable, il est possible que les travaux de réparation cités plus haut n'aient été réalisés que pour amadouer les moines, sans doute profondément offensés par l'intrusion au cœur de leur monde d'un bâtiment laïque si provocant, ces moines qui, en tout cas, même quarante ans après l'acte de fondation du duc, n'étaient encore dans leur majorité ni normands ni bretons, mais francs ${ }^{77}$. Peu après, en 1009 , dans le premier acte en notre possession passé entre l'abbaye et un duc, les moines s'adressent à Richard II non comme " duc des Normands " mais comme " marquis de Neustrie ", signe qu'ils ne se sentaient pas encore attachés au duché lui-même ${ }^{78}$.

Cela ne changea qu'après 1015, quand Gunnor, veuve de Richard ${ }^{\mathrm{er}}$, fit au Mont une donation considérable qui inaugura un patronage plus général de l'abbaye par les membres de la famille ducale ${ }^{79}$, et fut suivie, enfin, par un déluge de dons fonciers de la part de l'aristocratie normande, grande et petite.

\section{Le sort du clergé aubertien}

Les bénédictins étaient arrivés au Mont contraints et forcés et en butte aux soupçons, avec un droit d'occupation discutable. Bien sûr, ce ne fut pas ainsi qu'un siècle plus tard les moines eux-mêmes présentèrent leur histoire dans l'Introductio, écrite vers $1080-1085^{80}$. Selon celle-ci, le duc Richard jugea nécessaire d'intervenir dans les affaires de l'île à cause du comportement honteux des chanoines et de leur refus obstiné de s'amender comme l'y exhortait le prince. Les chanoines engageaient au rabais de jeunes prêtres pour célébrer l'office à leur place, comme s'il s'agissait de mercenaires, pendant qu'eux-mêmes menaient une vie de débauche, consacrée aux orgies, à la chasse et autres plaisirs ${ }^{81}$.

Quels que soient les faits - et, en écrivant plus d'un siècle plus tard, il est peu probable que l'auteur ait su de quoi il retournait ${ }^{82}$ - la descrip-

76. Ibidem, p. 172-180.

77. Ceci est évident d'après les noms donnés dans la liste de 50 moines vivants débattue plus haut : KeATS-ROHAN, Katharine, "Testimonies... ", art. cit., p. 174.

78. VAn TORHoudt, Éric, Centralité et marginalité..., op. cit., p. 807-821.

79. Keats-Rohan, Katharine, Cartulary..., op. cit., chartes numéros 2-5, p. 76-83.

80 . On pense que la construction de l'église romane de l'abbaye, remarquable du point de vue de sa structure, a été achevée pendant les mêmes années. Il est probable que l'Introductio a été composée pour marquer l'événement (GANDY, George, "L'architecture du Mont Saint-Michel selon la tapisserie de Bayeux et les "Très riches Heures » du duc de Berry ", Revue de l'Avranchin et du Pays de Granville, 90, 2013, p. 283).

81. Introductio, vi.2.

82. En général, les historiens admettent au pied de la lettre les critiques des chanoines trouvées dans l'Introductio, mais on ne peut pas accepter comme preuve de décadence 
tion est stéréotypée, donnant une image de la vie libre et indisciplinée que n'importe quel moine noir de la fin du XI ${ }^{\mathrm{e}}$ siècle aurait soupçonné caractéristique de clercs séculiers, souvent mariés ${ }^{83}$. Hildebrand lui-même, le futur pape Grégoire VII, avait donné l'assaut au mode de vie des chanoines en 1059, quand le synode du Latran avait exhorté ces derniers à abandonner la propriété privée pour mener une vie commune, comme les moines ${ }^{84}$. Plus largement, l'Introductio était une " histoire du point de vue du vainqueur ", destinée à donner la meilleure impression possible d'un épisode embarrassant pour les bénédictins. L'auteur s'est préoccupé principalement de rehausser le rôle des ducs normands et des bénédictins en amenant le Mont-Saint-Michel à la prééminence dont il jouissait alors, et quand il en est venu à expliquer l'expulsion des clercs, les prédécesseurs des moines devaient évidemment être présentés sous un jour défavorable. D'un autre côté, l'auteur va fort loin pour nous assurer que le duc a imploré les chanoines de s'amender : il n'aurait agi contre eux qu'après l'échec de toute persuasion et une fois obtenu l'appui de l'Église, en l'occurrence celui de l'archevêque de Rouen et du pape. Ceci suggère que, dans les années 1080 encore, la réputation de Richard Ire était sans doute ternie par le soupçon communément admis qu'il avait agi avec son impétuosité habituelle.

Selon l'Introductio, la dernière offre faite aux chanoines par le duc leur fut transmise par un envoyé : mandans clericis ut aut sacri monachatus ordinem susciperent aut a loco exirent ${ }^{85}$. La plupart des érudits ont interprété le verbe susciperent comme indiquant aux clercs qu'ils devaient partir du Mont s'ils n'étaient pas disposés à " adopter " la règle monastique. Cependant, Pierre Bouet, l'un des historiens récents persuadés que les clercs furent autorisés à rester sur l'île, traduit l'expression comme une consigne demandant aux clercs " d'admettre la présence " des bénédictins, de les " accepter " parmi eux, ou bien de partir. Cette interprétation me semble un peu forcée. Elle s'oppose aussi à la tradition transmise par les moines au cours des siècles $^{86}$. De toute façon, le texte de l'Introductio énonce clairement que les clercs refusèrent collectivement l'offre et, " comme ils l'avaient projeté de

des allégations avancées dans une œuvre de propagande justificative composée plus de cent ans après l'événement.

83. MuSSET, Lucien, "Recherches sur les communautés de clercs séculiers en Normandie au XI ${ }^{\mathrm{e}}$ siècle ", Mémoires de la Société des Antiquaires de Normandie, 55, 1961, p. 13-19.

84. Morris, Colin, The Papal Monarchy. The Western Church from 1050 to 1250, Oxford, Clarendon Press, 1989, p. 74-78.

85. Introductio, VII.1.

86. Ainsi dans le compte rendu de Guillaume de Saint-Pair, un moine du Mont, composé vers 1156 : "Le pape lui [le duc Richard] ordonnait [...] de chasser du mont tous les chanoines " [v.1835-7] ; et plus tard, "Notre seigneur le duc vous donne à tous [...] l'ordre de choisir ce que vous voudrez : être moines ou quitter ce lieu immédiatement " [v.1893-7] (texte en ancien français rendu en français moderne par Catherine Bougy) : Guillaume De Saint-Pair, Le Roman du Mont Saint-Michel, Bougy, Catherine (éd.), Caen, Presses Universitaires de Caen, 2009, p. 200-203 (cf. Bouet, Pierre et Desbordes, Olivier, Chroniques latines..., op. cit., p. 212-213; et pour un traitement additionnel, voir ibidem, p. 163). 
faire, quittèrent le lieu et partirent pour des destinations diverses, chacun selon son gré87".

Seuls deux d'entre eux, raconte l'auteur, restèrent : Durand, qui, animé par son amour de saint Michel, devint le chapelain du nouveau monastère, et Bernier, un vieux fauteur de troubles acariâtre, qui prétendit être trop malade pour déménager ${ }^{88}$. L'Introductio nous dit que les bénédictins relogèrent Bernier et prirent soin de lui dans des locaux en dessous du monastère, où il décéda bientôt de ses infirmités. Comme Pierre Bouet lui-même le remarque, les responsabilités assumées par Durand en tant que chapelain des moines avaient été probablement étendues au rôle de gardien des reliques dans l'église, prenant soin des vêtements liturgiques et présidant au bon déroulement des cérémonies ${ }^{89}$; il a dû ainsi passer la plupart de la journée et dormir la nuit à l'intérieur de l'abbaye. Le texte de l'Introductio n'induit donc pas que les clercs déplacés se soient regroupés pour reformer leur communauté ailleurs sur l'île ${ }^{90}$.

C'est néanmoins aujourd'hui une opinion courante. Un fait a encouragé sa diffusion : une communauté de douze chanoines était certainement présente sur l'île en 1061, presque un siècle après l'arrivée des Normands. Il apparaît, cependant, que ces chanoines devaient leur présence sur le Mont à une lutte de pouvoir contemporaine entre l'abbaye et son évêque. Ce n'est qu'à partir des années 1060 que les évêques normands en général réussirent à établir leur hégémonie sur les abbayes de leurs diocèses ${ }^{91}$. L'évêque nommé à Avranches en 1060, Jean d'Ivry, était un puissant aristocrate : son père Raoul avait été le demi-frère de Richard II. Jean était un réformateur ecclésiastique actif qui deviendrait bientôt archevêque de Rouen $^{92}$. Avec lui, pour ce qui touchait aux droits de son office, on ne plai-

87. Introductio, VII. 1 : pari obstinatione monachilem ordinem refutantes, juxta quod praemeditaverunt, ut praedictum est, loco cedentes, ut cuique visum est, diversas abierunt in partes.

88. Il s'avéra quelques années plus tard que la cause principale de la réticence de Bernier à quitter son logement était qu'il avait caché le squelette de saint Aubert dans les combles, ayant l'intention de partir avec dès qu'il le pourrait. Il l'avait secrètement et nuitamment exhumé de sa tombe sous l'autel de l'église paroissiale de Saint-Pierre, au pied du Mont, probablement en 965, quand il devint évident que les bénédictins étaient sur le point de s'emparer du Mont-Tombe. L'histoire de l'ultime redécouverte du squelette et de sa translation par les moines devint le joyau des miracles de l'abbaye du début du $\mathrm{XI}^{\mathrm{e}}$ siècle (De translatione et miraculis beati Autbert dans BOUET, Pierre et DESBORDES, Olivier, Chroniques latines..., op. cit., p. 212-213 et 248-253).

89. Ibidem, p. 214 , note 43.

90. Pierre Bouet trouve notable le fait que, selon lui, l'Introductio nous dise que Durand et Bernier ont tous deux récupéré auprès des moines leurs biens personnels, ce qui peut impliquer qu'aucun d'eux ne rejoignit la communauté monastique et qu'ils auraient ainsi pu former le noyau d'une communauté de clergé reconstituée ailleurs au Mont. Mais en fait ceci n'est dit que du maladif Bernier (BouEt, Pierre et DEsBordes, Olivier, Chroniques latines..., op. cit., p. 163, cf. le texte lui-même : ibidem, p. 214-215).

91. PotTs, Cassandra, Monastic Revival..., op. cit., p. 19-20.

92. Pour sa carrière, voir ALLEN, Richard, " "A proud and headstrong man”: John of Ivry, bishop of Avranches and archbishop of Rouen, 1060-79 ", Historical Research, 83, 2010, p. $189-227$. 
santait pas. Il semble avoir choisi de s'attaquer au Mont-Saint-Michel au moment où l'autorité de l'abbé Renouf était la plus faible. Un conflit parmi les moines avait tout récemment conduit un certain nombre d'entre eux à déménager sur l'îlot de Tombelaine, à deux kilomètres et demi du Mont, par défi envers leur abbé ${ }^{93}$. La conséquence de cette suite d'événements fut une convention, souscrite en 1061 et rédigée évidemment par l'évêque Jean lui-même, qui mit fin, largement en faveur de l'évêque, à une longue liste de querelles de juridiction à propos du Mont lui-même et de ses possessions ailleurs ${ }^{94}$.

La convention révèle qu'il y avait alors douze chanoines, résidant sur l'île mais soumis à l'évêque (canonici omnes sub episcopo proprie sunt) et nommés par lui ${ }^{95}$. L'importance des chanoines pour l'évêque Jean est soulignée par le fait qu'ils sont le sujet des paroles qui concluent le texte et qui déclarent audacieusement : Beatus enim Aubertus... eos [les chanoines] instituit et de suo episcopio ecclesie quam construxit duas villas, Icium scilicet et Genecium, ad usum suum et illorum contulit. Littéralement interprété, le texte prétend que cette maison de chanoines avait été instituée par Aubert lui-même. Mais il semble que cette formule servit simplement de justification au fait que l'évêque essayait de garder un œil sur le Mont en y implantant ses propres clercs. Il n'a pas pu faire partir les moines, mais lui-même et l'abbé Renouf savaient bien qu'au moment de leur installation, les bénédictins s'étaient approprié des terrains destinés à subvenir aux besoins de leurs prédécesseurs cléricaux, et notamment des propriétés situées à Montitier (Icium) et à Genêts qui, comme la Revelatio le confirme, appartenaient auparavant au patrimoine épiscopal. La convention a tout fait pour suggérer qu'Aubert n'avait eu aucune intention d'aliéner entièrement ces propriétés en faveur de ses clercs ${ }^{96}$. C'est ainsi que l'évêque Jean put exiger que les moines consacrent de nouveau des revenus de leurs terres à l'entretien d'un établissement de chanoines sur l'île.

Ces chanoines du xi ${ }^{\mathrm{e}}$ siècle ne résidaient pas dans le monastère; ils desservaient plutôt l'église paroissiale Saint-Pierre dans le village situé en contrebas $^{97}$. Mais il est évident qu'ils vivaient aux frais de l'abbaye, recon-

93. LAPORTE, Jean, « L’Abbaye du Mont Saint-Michel », dans LAPORTE Jean (dir.), Millénaire Monastique, t. 1, p. 78. Vers 1063, les mécontents furent recrutés par Odon de Bayeux pour refonder l'abbaye de Saint-Vigor, à côté de cette ville, d'où leur prieur (et par la suite, abbé) Robert de Tombelaine continua d'admonester son ancien abbé, Renouf du Mont Saint-Michel. Cependant, la propre disgrâce d'Odon entraîna la fermeture de SaintVigor en 1082 (Quivy, P. et THIRON, Jean, "Robert de Tombelaine et son commentaire sur le Cantique des cantiques ", dans ForEviLle, Raymonde [dir.], Millénaire Monastique, t. 2, p. 350-351 et GAZEAU, Véronique, Normannia monastica..., op. cit., t. 2, p. 5-6).

94. Le texte de la convention est reproduit et discuté dans BouET, Pierre et DESBORDES, Olivier, Chroniques latines..., op. cit., p. 375-378.

95. En se réservant le droit d'assigner les prébendes à chaque vacance, l'évêque contrôlait les nominations.

96. Revelatio, VII, 2.

97. En 1179-1181 on disait qu'ils desservaient les " autels paroissiaux de l'abbaye " : Chronique de Robert de Torigni, Deliste, Léopold (éd.), Rouen, Société de l'Histoire de 
naissance tacite que les bénédictins avaient injustement exproprié leurs prédécesseurs. On ne connaît pas la date précise de leur installation, mais ce fut certainement après 1020, Richard II ayant accordé à l'abbaye en 10221026 le droit exclusif de nommer les prêtres de l'église Saint-Pierre ${ }^{98}$. Selon toute vraisemblance, la reconnaissance, en 1061, que les moines avaient cédé ce droit à leur évêque était un des motifs de la convention, les chanoines s'étant installés juste après la nomination de Jean d'Ivry à Avranches en 1060. Le cadre de la convention concernait toutes les dimensions des pouvoirs respectifs de l'abbé et de l'évêque, mais l'un de ses buts paraît être d'avoir voulu implanter les chanoines dans le tissu de la vie montoise avant que les moines puissent trouver un moyen de les fragiliser.

Cependant, un siècle plus tard, dans les années 1170, l'un des plus puissants abbés de l'histoire du Mont, Robert de Torigni, se fit justice lui-même. Unilatéralement, il prit des mesures pour réduire à deux le nombre de chanoines et par la suite, sans consulter son évêque, il obtint l'approbation du pape Alexandre III conférant une légitimité rétrospective à son intervention. Le pape fit sa propre enquête auprès d'un évêque d'une province voisine et s'assura que les terres destinées à entretenir les chanoines ne suffisaient pas à plus de trois personnes. De ce fait, on racontait que, jusqu'à l'intervention de Robert de Torigni, les chanoines importunaient les pèlerins en mendiant des aumônes, nuisant à la réputation de l'abbaye ${ }^{99}$.

Il y a toutefois une autre façon de considérer cette histoire : l'appauvrissement des chanoines suggère que les moines n'étaient pas disposés à les entretenir sur les revenus généraux de l'abbaye, une des plus riches du nord-ouest de la France, et souhaitaient s'en débarrasser. Avec Robert de Torigni, ils avaient enfin un abbé qui disposait de relations avec le roi et la hiérarchie de l'Église beaucoup plus étroites que l'évêque et qui pouvait donc parvenir à ses fins.

Pour intéressante que soit leur histoire, les chanoines de 1061 n'avaient aucun lien institutionnel avec le clergé rencontré par les Normands quand ils arrivèrent au Mont en 965. L'Introductio, un texte écrit seulement une vingtaine d'années après la rédaction de la convention, donne un récit très clair de l'expulsion des clercs. La version des événements racontée par l'Introductio aurait été trop discordante avec le récit dont les chanoines de Saint-Pierre auraient pu régaler les pèlerins du Mont s'ils avaient vraiment été les héritiers des clercs d'Aubert. De même, quand vers 1156, le moine montois Guillaume de Saint-Pair écrivit son histoire de l'abbaye en vers vernaculaires pour divertir et informer ses visiteurs, il reprit l'histoire selon laquelle tous les chanoines, sauf deux, avaient quitté l'île, tout en ajoutant :

Normandie, t. 2, 1873, p. 312-313. Comme aucun autel désigné ainsi n'a pu être identifié dans l'abbaye, il doit s'agir de l'église du village.

98. Keats-Rohan, Katharine, Cartulary..., op. cit., numéro 2, p. 76-79.

99. Delisle, Léopold (éd.), Chronique..., op. cit., t. 2, p. 312-313. 
Après avoir quitté le Mont, les chanoines s'en allèrent où bon leur sembla, chacun de son côté, à sa guise. Que Dieu, le fils de Marie, leur vienne en aide, car je n'en sais pas plus sur le reste de leur vie ${ }^{100}$.

Écrire dans de tels termes aurait été absurde si, au temps de Guillaume, la communauté cléricale fondée par saint Aubert desservait toujours l'église Saint-Pierre pour les pèlerins et les villageois, à peine une centaine de mètres sous les murs de l'abbaye. Il est aussi peu probable que l'abbé historien contemporain Robert de Torigni ait pu ainsi déshonorer la mémoire d'Aubert, père fondateur de la communauté, au point d'entériner une déformation de la vérité par son moine Guillaume ou d'intervenir directement pour réduire les chanoines de Saint-Pierre à l'indigence et à un nombre dérisoire en sachant que ceux-ci étaient les derniers représentants de la première communauté religieuse du VIII ${ }^{\mathrm{e}}$ siècle.

Il est donc clair que les clercs d'Aubert furent expulsés et dispersés dans le monde par Richard I ${ }^{\text {er }}$ en 965 . Au moment de leur suppression, il semble que la communauté était toujours prospère, car ils avaient totalement reconstruit l'église Saint-Michel deux ou trois décennies auparavant ${ }^{101}$. Cette preuve de la popularité ininterrompue du pèlerinage au Mont peut aussi soutenir la thèse importante de Van Torhoudt pour qui l'absence d'épiscopat local au cours du $\mathrm{x}^{\mathrm{e}}$ siècle n'entraîna pas la disparition complète du réseau de clergé paroissial dans le Cotentin, d'où provenait une partie importante des pèlerins ${ }^{102}$. Il est aussi évident que, dès 965 , Richard $\mathrm{I}^{\text {er }}$ put intervenir dans le "Far West " du duché, même si l'absence de dons en faveur de son monastère laisse planer le doute sur le degré de sa mâ̂trise de la région.

100. Guillaume de Saint-Pair, Roman..., op. cit., p. 202-205.

101. GANDY, George, "Who built what...? ", art. cit., p. 159-161.

102. VAn ToRHOUDT, Éric, Centralité et marginalité..., op. cit., p. 199-200, 389-390 et 765-766. 


\section{RÉSUMÉ}

Le point de vue traditionnellement admis selon lequel l'abbaye du Mont Saint-Michel fut fondée vers 965 par le duc normand Richard I a été largement remis en question au cours des dernières décennies car il constitue une narration normande, écrite a posteriori dans un but apologétique. Que Richard ait contrôlé la partie Ouest de son duché à une date si précoce, si toutefois il l'a contrôlée un jour, condamne cette interprétation. D'autres hypothèses ont été avancées, par exemple que des bénédictins bretons aient précédé l'arrivée des Normands et que ceux-ci, de toute façon, ne se seraient pas beaucoup intéressés à l'îlot avant le début du $\mathrm{XI}^{\mathrm{e}}$ siècle. Cet article examine ces nouvelles théories et montre qu'elles sont peu convaincantes. L'auteur soutient que la communauté de clercs installée par l'évêque Aubert d'Avranches vers 709 était toujours responsable de ce lieu de pèlerinage au milieu du $\mathrm{x}^{\mathrm{e}}$ siècle; que Richard Ier les a remplacé par des bénédictins en 965 faisant ainsi un geste politique afin d'obtenir une paix avec l'empereur Otton ${ }^{\text {er }}$, le roi franc Lothair et le comte de Blois, Thibaud; qu'au même moment il a expulsé les clercs; et que, par la suite, il a ignoré sa nouvelle abbaye qui, par conséquent, a dû lutter pendant une génération avant d'être bien établie.

\section{ABSTRACT}

The traditional belief that the abbey of Mont Saint-Michel was founded in c.965 by the Norman duke Richard I has been widely questioned in recent decades. It has been represented as essentially an ex post facto and self-serving Norman narrative, the veracity of which is put in doubt by the improbability (it is said) that Richard exercised any control over the west of his duchy at such an early date, if he ever did. Alternative possibilities have been promoted, such as that Breton benedictines preceded the Normans and that the latter did not even take much interest in the island until early in the $11^{\text {th }}$ century. This article re-examines the new orthodoxies and finds them unpersuasive. It argues that the community of clerks installed in c.709 by Bishop Aubert of Avranches was still in charge of the pilgrimage shrine in the mid-10 $0^{\text {th }}$ century; that Richard I replaced them with benedictines in 965 as a political gesture, to help secure a peace with the Emperor Otto the Great, the Frankish king Lothar and Count Thibaud of Blois; that he simultaneously expelled the clerks and thereafter ignored his new abbey, which consequently struggled for a generation to become established. 